\title{
Probing spin configurations in quantum dots
}

M. C. Rogge, C. Fühner, and R. J. Haug

Citation: AIP Conference Proceedings 893, 809 (2007); doi: 10.1063/1.2730137

View online: https://doi.org/10.1063/1.2730137

View Table of Contents: http://aip.scitation.org/toc/apc/893/1

Published by the American Institute of Physics

\section{Conference Proceedings}

\section{Get $30 \%$ off all print proceedings!}

Enter Promotion Code PDF30 at checkout 


\title{
Probing spin configurations in quantum dots
}

\author{
M. C. Rogge, C. Fühner and R. J. Haug \\ Institut für Festkörperphysik, Leibniz Universität Hannover, Appelstraße 2, 30167 Hannover, Germany
}

\begin{abstract}
We study the magnetoconductance of lateral quantum dots in high magnetic fields. We use spin blockade and Kondo effect to investigate the spin configuration and the spin filling mechanism and find a dependence on the electron number of the quantum dot.
\end{abstract}

Keywords: quantum dots, Coulomb blockade, spin blockade, Kondo effect PACS: 73.63.Kv, 73.23. Hk, 72.15.Qm, 73.21.La

Spin blockade [1] and Kondo effect [2] in lateral quantum dots [3] have been studied intensively throughout the last years. The spin blockade induces spin dependent transport due to spin polarized edge channels in the electron reservoirs in perpendicular magnetic fields. As these channels are spatially separated the wave function overlap between the dot and the reservoirs and thus the tunneling probability depends on the spin of the tunneling electron. Spin up transport is suppressed compared to spin down transport leading to high or low conductance along Coulomb peaks. The Kondo effect enables cotunneling processes when sequential tunneling is Coulomb blocked $[4,5]$ and depends on the spin configuration of the dot. In a perpendicular magnetic field a periodic pattern, so called chessboard pattern, is established showing alternating low and high Kondo conductance in Coulomb valleys $[6,7]$.

Very recently we have shown that both effects can be combined in intermediate magnetic fields [8]. While the spin blockade reveals the spin of the tunneling electron, the Kondo effect yields information about the spin configuration in Coulomb blocked regions. Both effects combined show the full information about the spin configuration and the spin filling of dot orbitals. As both effects show the same periodicity as a function of a magnetic field only two combinations are possible. A strong peak conductance can be combined either with a strong or weak valley conductance in the next Coulomb valley at higher energy. We observed both combinations in different samples depending on the electron number. For low electron numbers we found a strong peak with a strong valley conductance reflecting an unpolarized spin configuration and a regular filling of dot orbitals [9]. The opposite combination was found in a second sample with higher electron number. Therefore a polarized spin configuration was assumed [8]. A third sample [10] revealed several regions of different combinations showing changes of the spin configuration with increasing elec-

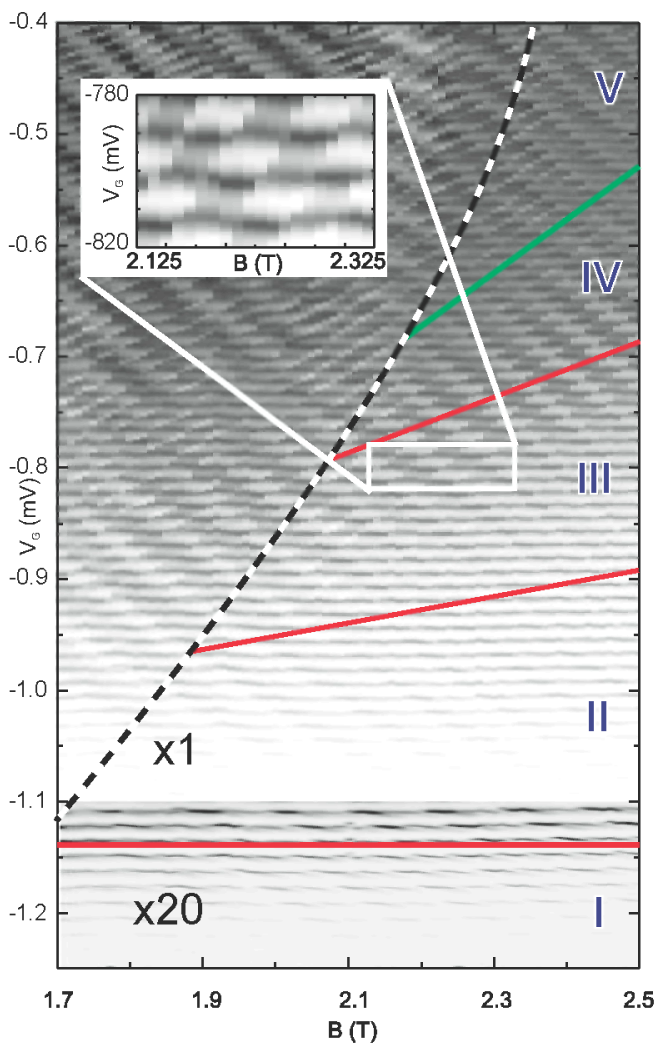

FIGURE 1. Magnetoconductance of a lateral quantum dot showing a combined pattern of spin blockade and Kondo conductance (dark for high conductance, bright for low). Five regions of different combinations are found. The transitions are marked with solid lines

\section{tron number [11]}

Here we want to focus on the transitions between the different configurations in the third sample.

Figure 1 shows the magnetoconductance of this sample with both Kondo effect and spin blockade. Right of the dashed line a regular chessboard pattern is observed 

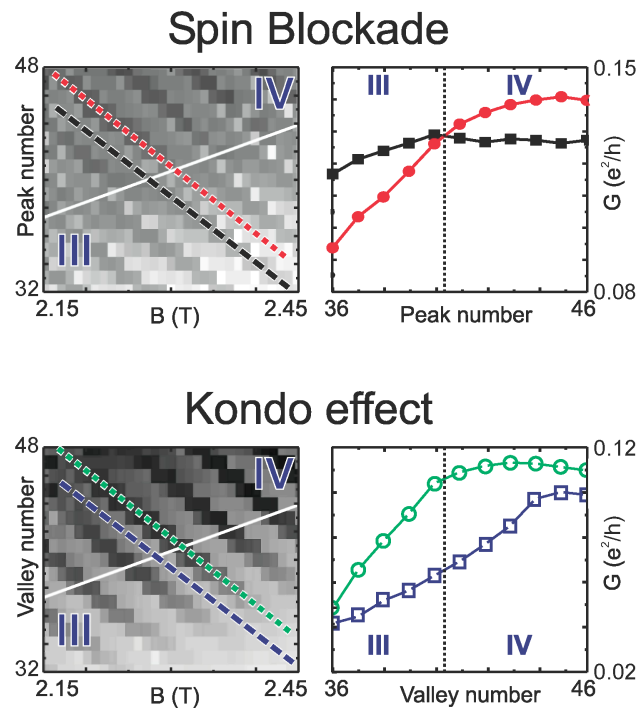

FIGURE 2. At the transition from III to IV spin blockade (top) and Kondo effect (bottom) are analyzed separately by plotting either the peak conductance or the valley conductance as a function of peak (valley) number and magnetic field (left). Traces along dashed and dotted lines at the transition show a crossing of the spin blockade pattern and no change of the Kondo pattern (right)

which is shown in more detail in the inset. As a function of the magnetic field we find a periodic modulation of the peak amplitude of Coulomb peaks as well as a strong alternation of the Kondo conductance in the valleys between the peaks. The inset shows a strong Kondo conductance above a suppressed peak. The complete measurement covers five regions of different combinations. The transitions are marked with solid lines.

We now focus on the transition between regions III and IV. To investigate this transition we study spin blockade and Kondo effect separately. This is done in Fig. 2. To investigate the spin blockade we plotted the peak amplitudes as a function of the peak number and the magnetic field (left side of Fig. 2). The resulting graph is comparable with the original measurement without the Coulomb valleys. The periodicity of the peak amplitude is now visible as a pattern of lines with negative slope. Strong lines (dark) show spin down transport, weak lines (bright) show spin up transport. If we follow these lines from the lower right to the upper left we find that strong lines are changed into weak lines when crossing the transition and vice versa. Traces along two lines are shown on the right. Circles reflect the conductance along the dotted trace, squares along the dashed trace. A clear crossing is visible. Therefore the transition between regions III and IV is induced by a change of the spin blockade pattern reflecting a change in the spin filling mechanism. The same analysis was done for

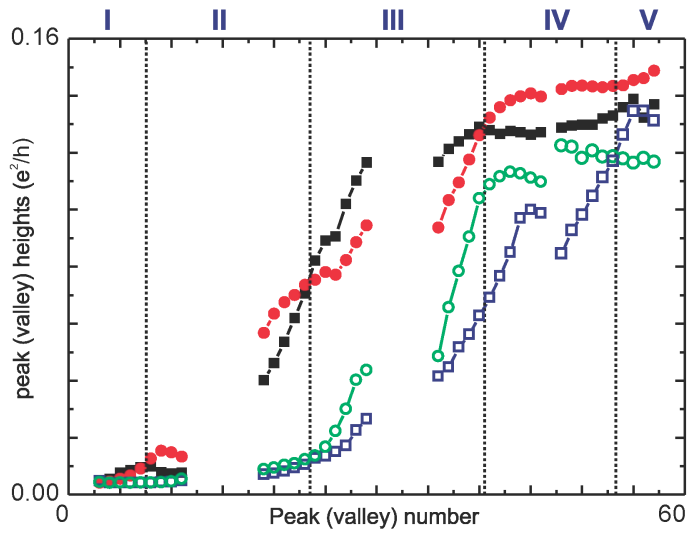

FIGURE 3. Analysis of all transitions as in Fig. 2. The first three transitions show a change of the spin blockade, the last transition a change of the Kondo conductance.

the Coulomb valleys (lower part of Fig. 2). The plot on the left can be compared with the original measurement without the Coulomb peaks. Again we find a pattern of lines with negative slopes. In contrast to the spin blockade pattern there is no change of this pattern at the transition as illustrated by the traces on the right.

This analysis was done for all transitions. The result is plotted in Fig. 3. The first three transition show a change of the spin blockade pattern and no significant change of the Kondo conductance while the last transition shows the opposite behaviour.

In conclusion we have investigated transitions of the combined Kondo-spin blockade pattern of a lateral quantum dot in high magnetic fields. We found four transitions revealing changes of the spin configuration. Detailed analysis of these transitions have shown that only one part of the pattern, either the spin blockade pattern or the Kondo pattern, is responsible for each transition.

This work has been supported by BMBF.

\section{REFERENCES}

1. M. Ciorga et al., Phys. Rev. B 61, R16315 (2000).

2. J. Kondo, Prog. Theor. Phys. 32, 37 (1964).

3. L. P. Kouwenhoven et al., in Mesoscopic Electron Transport, Kluwer, Dordrecht 345 series E, 105-214 (1997).

4. L. I. Glazman, and M. E. Raikh, JETP Lett. 47, 452 (1988).

5. T. K. Ng, and P. A. Lee, Phys. Rev. Lett. 61, 1768 (1988).

6. M. Stopa et al., Phys. Rev. Lett. 91, 046601 (2003).

7. M. Keller et al., Phys. Rev. B 64, 033302 (2001).

8. D. Kupidura et al., Phys. Rev. Lett. 96, 046802 (2006).

9. M. C. Rogge et al., Physica Status Solidi, Quantum Dot 2006 proceeding (to be published).

10. C. Fühner et al., Phys. Rev. B 66, R161305 (2002).

11. M. C. Rogge et al., cond-mat/0606414 (2006). 